\title{
Association of ANRIL gene single- nucleotide polymorphisms with Allergic rhinitis in Kurdish population from Kermanshah, Iran
}

\section{Sara Falahi}

Kermanshah University of Medical Sciences

\section{Parisa Feizolahi}

Kermanshah University of Medical Sciences

Ali Monshizadeh

Legal Medicine Organization

\section{Zahra Mahmoudi}

Kermanshah University of Medical Sciences

\section{Jila Mahdavi}

Payame Noor University

\section{Farhad Salari}

Kermanshah University of Medical Sciences

\section{Ali Gorgin Karaji}

Kermanshah University of Medical Sciences

Alireza Rezaiemanesh ( $\sim$ alireza.rezaiemanesh@kums.ac.ir)

Kermanshah University of Medical Sciences

\section{Research Article}

Keywords: Allergic rhinitis, ANRIL IncRNA gene, Single nucleotide polymorphism

Posted Date: March 3rd, 2022

DOI: https://doi.org/10.21203/rs.3.rs-1401694/v1

License: (c) (1) This work is licensed under a Creative Commons Attribution 4.0 International License. Read Full License 


\section{Abstract}

Background: Allergic rhinitis (AR) is the most common inflammatory disorder of the upper airway caused by aberrant immune responses to allergens in genetically predisposed individuals. Recently, the long noncoding RNA (IncRNA) antisense non-coding RNA in the INK4 locus (ANRIL) has been identified as a novel genetic factor associated with increased AR risk. This study aimed to evaluate the potential correlation of $A N R I L$ gene single nucleotide polymorphisms (SNPs) with AR risk in the kurdish population of Kermanshah, Iran.

Methods: In this case-control study, 130 AR patients and 130 healthy controls were recruited to genotype for two SNPs of the ANRIL gene (rs1333048, rs10757278) using the Tetra-primer amplification refractory mutation system PCR (T-ARMS-PCR) method.

Results: Our results showed no significant difference for the alleles and genotypes frequency distribution of IncRNA ANRIL SNPs (rs1333048, rs10757278) between AR patients and healthy controls ( $p>0.05)$. Additionally, the dominant, additive, and recessive genetic models of both SNPs were not associated with altered susceptibility to AR risk ( $p>0.05)$.

Conclusion: The results demonstrated that the ANRIL gene rs1333048 and rs10757278 polymorphisms might not be associated with susceptibility to AR in the kurdish population of Kermanshah, Iran

\section{Introduction}

Allergic rhinitis (AR) is a chronic inflammatory disease of the nasal mucosa characterized by nasal rhinorrhea, sneezing, itching, and congestion. Epidemiological studies have reported that the prevalence of AR is $10-30 \%$ of adults and up to $40 \%$ of children worldwide (Mims, 2014; Wheatley and Togias, 2015). The characteristic inflammation in AR is induced by lgE-mediated reactions against the inhaled allergens, during which different inflammatory cells, including helper T (Th) 2 and Th17 cells, mast cells, basophils, and eosinophils are activated. Activation of the immune cells during AR results in the production of a variety of proinflammatory cytokines and mediators, such as interleukin (IL)-13, IL-4, IL-5, IL-17, tumor necrosis factor (TNF)-a, histamine, and leukotrienes, contributing to the increased inflammation of AR (Varshney and Varshney, 2015; Wheatley and Togias, 2015). In addition to environmental exposures, genetic predisposition and epigenetic modifications might be involved in the dysregulation of the immune system in AR (DeVries and Vercelli, 2015; Kabesch and Tost, 2020).

Emerging evidence has placed a new emphasis on the role of long non-coding RNAs (IncRNAs) in regulating immune responses and the pathogenesis of several immune-related disorders, such as allergic diseases (Flores-Concha and Oñate, 2020; Ghafouri-Fard et al., 2020; Zuo and Xu, 2020). LncRNAs are a class of RNAs containing more than 200 nucleotides in length with having no or very low protein-coding potential (Gao et al., 2020). Based on findings from other studies, IncRNAs can participate in epigenetic regulation of gene expression at transcriptional, post-transcriptional, translation, and post-translational levels through interacting with DNA, mRNAs, miRNAs, and proteins. Furthermore, LncRNAs also contribute 
to a variety of biological processes, including embryonic development, cell differentiation, and metabolism (Gao et al., 2020; Zhang et al., 2019).

Antisense non-coding RNA in the INK4 locus (ANRIL), as one of the earliest discovered IncRNAs, plays an important role in the development and progression of inflammation-related diseases, such as systemic lupus erythematosus (SLE), cardiovascular diseases (CAD), diabetes, and cancers (Abd-Elmawla et al., 2018; Chen et al., 2019; Mehta-Mujoo et al., 2019; Qin and Liu, 2019). The ANRIL gene is located in the chromosome 9p21 region, and it has been identified as a novel genetic factor associated with the pathogenesis of AR (Qian et al., 2019). According to a recent study, IncRNA ANRIL expression in the nasal mucosa of AR patients was upregulated compared to controls, and it was positively correlated with increased AR risk, severity, and inflammation. (Qian et al., 2019). More interestingly, IncRNA ANRIL expression was discovered to be positively correlated with increased TNF-a, IL-4, IL-6, IL-13, and IL-17, whereas it was negatively correlated with interferon (IFN)- $y$ and IL-10 mRNA expressions, suggesting that it was related to elevated inflammation of AR (Qian et al., 2019).

In recent years, given the important role of IncRNA ANRIL in the pathogenesis of various inflammatory diseases, single nucleotide polymorphisms (SNP) of the ANRIL gene have gained widespread attention (Lu et al., 2020; Mollahosseini et al., 2020). Although the role of ANRIL in the regulation of immune responses and expression of some AR-related factors has been acknowledged, the participation of ANRIL genetic variants in AR has not been assessed yet. Genetic variants of the ANRIL gene may act as a risk or protective factor for AR development. Regarding the indispensable contribution of LncRNA underlying inflammatory disease, this is the first study, aimed to evaluate the role of ANRIL gene polymorphisms with AR susceptibility in the kurdish population of Kermanshah, Iran.

\section{Materials And Methods}

Study area and population

The study population consisted of 130 AR patients and 130 age- and gender-matched healthy controls. All samples were chosen from the Kurdish population of Kermanshah province, located in the west of Iran. Kurds, the most ancient indigenous people, lived mainly in the Zagros Mountains for thousands of years with owning their sociocultural values. To note, only the Kurdish population were included in this study, and participants with other ethnicities were excluded.. All patients with AR were diagnosed by an allergist according to diagnostic criteria described by the Practical Guideline for the management of allergic rhinitis (Wang et al., 2018). Healthy controls were collected from blood donors that did not have any history of inflammatory, allergic, and autoimmune disorders. The study protocol was approved by the ethics committee of Kermanshah University of Medical Sciences (IR.KUMS.REC.1399.1008). After being informed of the study and obtaining written consent from all study participants, blood sampling was conducted.

\section{DNA extraction and SNP genotyping}


The genomic DNA samples from peripheral blood of the patients and control subjects were extracted by the salting-out method. The purity and concentration of the extracted DNA samples were determined by the NanoDrop 2000-UV-Vis spectrophotometer (Thermo Scientific, USA).

Genotype determination for selected SNPs in the ANRIL gene (rs1333048, rs10757278) was performed by the tetra-primer amplification refractory mutation system PCR (T-ARMS-PCR) method. The specific primers for both SNPs were designed by PRIMER1 online tool (http://primer1.soton.ac.uk/primer1.html). The location of the SNPs and primers sequence are shown in Table 1.

Table 1

The sequence of primers and product size for T-ARMS PCR.

\begin{tabular}{|c|c|c|c|}
\hline Polymorphism & Primers & Primer sequence $\left(5^{\prime}-3^{\prime}\right)$ & Product size (bp) \\
\hline \multirow[t]{4}{*}{ rs1333048 A >C } & $\mathrm{FI}$ (A allele) & TTCATGCTATTTTGAGGAGATGTCTA & \multirow{4}{*}{$\begin{array}{l}\text { A allele: } 185 \\
\text { C allele: } 253 \\
\text { Outer primers: } 382\end{array}$} \\
\hline & RI (C allele) & TTTTATAAATATTTCAATAATTCGACACTG & \\
\hline & FO & TGCTTGATAACCAATTTTATTTGTTAG & \\
\hline & RO & TTCAACTGTTGATCATATGGTTAGTATG & \\
\hline \multirow[t]{4}{*}{ rs10757278 A > G } & $\mathrm{FI}$ (A allele) & AAGTCAGGGTGTGGTCATTCCGGGAA & \multirow{4}{*}{$\begin{array}{l}\text { A allele: } 263 \\
\text { G allele: } 234 \\
\text { Outer primers: } 443\end{array}$} \\
\hline & RI (G allele) & АСTACTCTGTCTTGATTCTGCATCGCTTCC & \\
\hline & FO & GGGCATTAAGAAAGGGATGGGTAGACAAAA & \\
\hline & RO & GCTGTTCCCAAGTAGCCAGGACTACCTCT & \\
\hline
\end{tabular}

The PCR reactions were carried out in microtubes with a total volume of $15 \mu \mathrm{l}$, containing $0.5 \mu \mathrm{l}$ of genomic DNA as a template, $0.7 \mu \mathrm{l}$ of each inner primer, $0.3 \mu \mathrm{l}$ of each outer primer, $7 \mu \mathrm{l}$ of multiplex PCR master mix, and $5.5 \mu \mathrm{l}$ of DNAase free distilled water. PCR amplification was performed with an initial denaturation at $95^{\circ} \mathrm{C}$ for 5 minutes, followed by 35 cycles of $95^{\circ} \mathrm{C}$ for 45 seconds, annealing for 30 seconds at $52^{\circ} \mathrm{C}$ for rs 1333048,30 seconds at $60^{\circ} \mathrm{C}$ for rs 10757278 , and extension for 55 seconds at $72^{\circ} \mathrm{C}$, with a final extension of $72^{\circ} \mathrm{C}$ for 5 minutes. The amplified products were electrophoresed on $2 \%$ agarose, stained with safe stain. According to the agarose gel electrophoresis for rs10757278 $A>G$, the AA genotype was represented if two fragments with $443 \mathrm{bp}$ and $262 \mathrm{bp}$ were generated, the AG genotype was detected in three fragments with 443bp, 262bp and 236bp, and the GG genotype was identified if two fragments with 443bp and 236bp were generated. Based on the agarose gel electrophoresis for rs $1333048 \mathrm{~A}>\mathrm{C}$, the AA genotype produced two fragments of $381 \mathrm{bp}$ and $184 \mathrm{bp}$, the AC genotype yield three fragments of $381 \mathrm{bp}, 252 \mathrm{bp}$ and $184 \mathrm{bp}$, and the CC genotype produced two fragments of $381 \mathrm{bp}$ and 252bp.

\section{Statistical analysis}


Comparison of genotype and allele distribution between case and controls were analyzed by Chi-square test, and the relation of genotypes with AR risk was estimated by calculating odds ratios (ORs) and their $95 \%$ confidence intervals $(\mathrm{Cl})$ using logistic regression analyses. The web-based SHEsis software (version 4.2) was used for the analysis of pairwise linkage disequilibrium and haplotype structure. The accordance of genotype frequencies with Hardy-Weinberg Equilibrium (HWE) was performed using the Chi-square test. Differences with a $P$ value less than 0.05 were considered as statistically significant. Statistical analyses were performed using the SPSS software version 23 (SPSS, Chicago, IL, USA).

\section{Results}

\section{Demographic characteristic}

The study population consisted of 130 AR patients, involving 60 males $(46.15 \%)$ and 70 females (53.85\%) and 130 control subjects, containing 60 males (46.15\%) and 70 females (53.85\%). The mean age of AR patients and control group was $35.81 \pm 10.75$ and $35.92 \pm 10.45$, respectively. There were no statistically significant differences between the two groups of AR patients and healthy controls for age and gender $(P>0.05)$, indicating that the study groups were matched regarding age and gender.

\section{Allele and genotype frequencies of SNPs}

The genotype distribution and allele frequencies of the two investigated SNPs, rs1333048 and rs 10757278 in ANRIL gene in healthy controls and AR patients are summarized in Table 2. Distribution of genotype frequencies of rs1333048 (but not rs10757278) was in accordance with HWE in both patient and controls groups. 
Table 2

Distribution of allele and genotype frequencies for ANRIL gene polymorphisms in patients with AR and controls.

\begin{tabular}{|lllll}
\hline SNPs/Genetic models & $\mathrm{AR}(\mathrm{N}=130)$ & Control $(\mathrm{N}=130)$ & Pvalue & OR $(95 \% \mathrm{Cl})$ \\
\cline { 2 - 3 } & $\mathrm{n}(\%)$ & $\mathrm{n}(\%)$ & &
\end{tabular}

rs10757278 A>G

Allele frequency

$\begin{array}{lllll}\text { A } & 102(39.2 \%) & 87(33.5 \%) & 0.17 & 1.284(0.897-1.837) \\ \text { G } & 158(60.8 \%) & 173(66.5 \%) & & \text { Reference }\end{array}$

Genotype frequency

$\begin{array}{lllll}\text { AA } & 6(4.6 \%) & 2(1.5 \%) & 0.08 & 3.971(0.754-20.906) \\ \text { AG } & 90(69.2 \%) & 83(63.8 \%) & 0.18 & 1.435(0.840-2.453) \\ \text { GG } & 34(26.2 \%) & 45(34.6 \%) & & \text { Reference }\end{array}$

Dominant model

$\begin{array}{lllll}\text { AA + AG } & 96(73.8 \%) & 85(65.4 \%) & 0.13 & 1.495(0.878-2.546) \\ \text { GG } & 34(26.2 \%) & 45(34.6 \%) & & \text { Reference }\end{array}$

Additive model

\begin{tabular}{|lllll|}
\hline AG & $90(69.2 \%)$ & $83(63.8 \%)$ & 0.35 & $1.274(0.760-2.136)$ \\
\hline AA + GG & $40(30.8 \%)$ & $47(36.2 \%)$ & & Reference \\
\hline Recessive model & & & & \\
\hline AA & $6(7 \%)$ & $2(7.7 \%)$ & 0.15 & $3.097(0.613-15.637)$ \\
\hline AG + GG & $124(93.07 \%)$ & $128(92.3 \%)$ & & Reference \\
\hline rs1333048 A $>$ C & & & & \\
\hline Allele frequency & & & & \\
\hline A & $131(50.4 \%)$ & $134(51.5 \%)$ & 0.79 & Reference \\
\hline C & $129(49.6 \%)$ & $126(48.5 \%)$ & & Reference \\
\hline Genotype frequency & & & & $0.743(0.415-1.332)$ \\
\hline AA & $39(30 \%)$ & $35(26.9 \%)$ & & \\
\hline AC & $53(40.8 \%)$ & $64(49.2 \%)$ & 0.31 & $0.477)$ \\
\hline
\end{tabular}

SNP, Single Nucleotide Polymorphism; AR, Allergic Rhinitis; OR, Odds Ratio; Cl, Confidence Interval 


\begin{tabular}{|c|c|c|c|c|}
\hline \multirow[t]{2}{*}{ SNPs/Genetic models } & $A R(N=130)$ & Control $(N=130)$ & \multirow[t]{2}{*}{$P$ value } & \multirow[t]{2}{*}{ OR $(95 \% \mathrm{Cl})$} \\
\hline & n (\%) & $\mathrm{n}(\%)$ & & \\
\hline $\mathrm{CC}$ & $38(29.2 \%)$ & $31(23.8 \%)$ & 0.77 & $1.100(0.570-2.125)$ \\
\hline \multicolumn{5}{|l|}{ Dominant model } \\
\hline$A C+C C$ & $91(70 \%)$ & $95(73.1 \%)$ & 0.58 & $0.860(0.501-1.474)$ \\
\hline AA & $39(30 \%)$ & $35(26.9 \%)$ & & Reference \\
\hline \multicolumn{5}{|l|}{ Additive model } \\
\hline$A C$ & $53(40.8 \%)$ & $64(49.2 \%)$ & 0.17 & $0.710(0.435-1.159)$ \\
\hline$A A+C C$ & $77(59.2 \%)$ & $66(50.8 \%)$ & & Reference \\
\hline \multicolumn{5}{|l|}{ Recessive model } \\
\hline $\mathrm{CC}$ & $38(31.7 \%)$ & $31(23.8 \%)$ & 0.16 & $1.480(0.848-2.584)$ \\
\hline$G G+A C$ & $82(68.3 \%)$ & 99 (76.2\%) & & Reference \\
\hline
\end{tabular}

The two SNPs were successfully genotyped for AR patients and healthy controls. Select T-ARMES-PCR gels for rs 10757278 and rs1333048 polymorphisms are shown in Fig. 1 and Fig. 2, respectively.

Our results revealed no significant differences for the allele and genotype frequencies of ANRIL SNPS (rs1333048, rs10757278) between AR patients and healthy controls $(P>0.05)$. In addition,the genetic models of dominant, additive, and recessive as well as haplotype frequencies showed no significant association with AR risk ( $P>0.05$; Table 2 and Table 3$)$.

Table 3

Distribution of haplotypes for ANRIL gene polymorphisms in AR patients and control group.

\begin{tabular}{|lllllll|}
\hline Haplotypes & Patients & Controls & $X^{2}$ & Pvalue & OR & $95 \%$ Cl \\
\hline A - A & $91.09(0.35)$ & $80.29(0.309)$ & 1.015 & 0.31 & 1.207 & $(0.831-1.741)$ \\
\hline A - C & $10.91(0.042)$ & $6.71(0.026)$ & 1.037 & 0.30 & 1.654 & $(0.622-4.396)$ \\
\hline G - A & $39.91(0.153)$ & $53.71(0.207)$ & 2.480 & 0.11 & 0.696 & $(0.443-1.094)$ \\
\hline G - C & $118.09(0.454)$ & $119.29(0.459)$ & 0.011 & 0.91 & 0.982 & $(0.695-1.386)$ \\
\hline OR, Odds Ratio; Cl, Confidence Interval & & & & \\
\hline
\end{tabular}

\section{Discussion}


In the current study, we investigated the association between the ANRIL gene polymorphisms, including rs 1333048 and rs10757278, with susceptibility to AR in the kurdish population of Kermanshah, Iran. Our results showed no significant association between these two polymorphisms and AR risk.

From an etiopathological point of view, AR is considered a complex multi-factorial inflammatory disease in which the genetic factors are critically involved in the activation of inflammatory responses (Broide, 2010). Although many studies have identified a large number of genetic factors involved in the pathogenesis of $A R$, the underlying mechanisms of gene dysregulation have not yet been identified. Recently, non-coding RNAs (ncRNAs), like microRNAs (miRNAs) and IncRNAs, as crucial regulators of gene expression, have been suggested to be involved in the regulation of immune responses and the pathogenesis of several immune-related disorders (Ghafouri-Fard et al., 2020; Mulrane et al., 2013; Rakhshan et al., 2020; Rezazadeh et al., 2018; Wei et al., 2017).

Regarding the nature of AR as Th2-mediated responses and the role of IncRNAs and miRNAs in the regulation of Th2 responses, no wonder that altered expression of these ncRNAs might contribute to the pathogenesis of AR. Intriguingly, the importance of miRNAs was determined in AR pathogenesis. For example, upregulation of miR-126-5p, miR-19a-5p, and miR-26a-5p was reported in the nasal mucosa of AR patients compared to healthy control (Rakhshan et al., 2020). Recently, a study indicated that IncRNA ANRIL expression in the nasal mucosa of AR patients was remarkably increased, and it was positively associated with upregulation of pro-inflammatory cytokines (IL-4, IL-6, IL-13, and IL-17) as well as disease severity such as itching and congestion. Hence, these observations suggest that the IncRNA ANRIL might be involved in the pathogenesis of AR (Qian et al., 2019). Although it is not clear how IncRNA ANRIL might play a role in regulating AR-related cytokines and miRNAs, it appears that the sponging of anti-inflammatory miRNAs, such as miR-Let7e and miR-18b by IncRNA ANRIL might be an explanation. Interestingly, the binding of ANRIL to an ANRIL binding transcriptional factor (Yin Yang 1) may be considered another mechanism, leading to upregulating IL- 6 and IL-8 expression in human endothelial cells. (Hori et al., 2017; Huo et al., 2016; Li et al., 2018; Qian et al., 2019). It is worth emphasizing that Signal transducer and activator of transcription 1 (STAT1) signaling contributed to ANRIL expression, which participates in the regulation of immune response through induction of the proinflammatory cytokine IFN-y (Harismendy et al., 2011). Given the association between nuclear factor (NF)-KB pathway and the crucial role of ANRIL in the expression of several pro-inflammatory genes regulated by NF-KB (Wee et al., 2017; Zhou et al., 2016), ANRIL might be a missing puzzle piece in the pathogenesis of AR. However, this speculation should be further investigated.

One of SNPs within the ANRIL gene has been reported to change the phenotypic traits and function of the genes, such as changes in the binding site for proteins involved in signaling pathways (Harismendy et al., 2011; Taheri et al., 2017). For instance, the polymorphism rs10757278 of the ANRIL gene has been demonstrated to disturb the binding of the STAT1 (Harismendy et al., 2011). In recent years, different studies have demonstrated the association of SNPs of ANRIL gene with the susceptibility to different diseases(Hariri et al., 2020; Rezazadeh et al., 2018; Taheri et al., 2017). 
Taheri and colleagues revealed that the GG genotype of rs10757278 and AA genotype of rs1333048 A>C were associated with prostate cancer and benign prostate hyperplasia (BPH) risk in an Iranian population (Taheri et al., 2017). Besides, rs 10757278 was significantly associated with coronary artery disease (CAD) risk in patients from Iraq, Iran, and Poland (Hariri et al., 2020; Niemiec et al., 2012; Suleiman et al., 2019). Additionally, ANRIL gene rs 10757278 and rs1333048 SNPs were shown to confer a risk for psoriasis development in an Iranian population (Rakhshan et al., 2020). In accordance with our study, there was no significant association of ANRIL gene rs1333048 and rs 10757278 SNPs with both multiple sclerosis (MS) and breast cancer (Khorshidi et al., 2017; Rezazadeh et al., 2018). However, haplotype analysis of $A N R / L$ gene polymorphisms (rs1333045, 1333048, rs4977574, and rs10757278) indicated a protective effect of CCGG and TAAA haplotypes in MS while TAGG and CCGA haplotypes were significantly associated with increased risk of MS (Rezazadeh et al., 2018). Moreover, haplotype analysis (with an order of rs1333045, 1333048, rs4977574, and rs10757278 SNPs) demonstrated that TCGA haplotype was associated with breast cancer risk (Khorshidi et al., 2017). The discrepancy in the reports concerning the contribution of $A N R I L$ polymorphisms to different diseases might stem from populationspecific genetic stratification, sample size, different genotyping methods, and the involvement of other genetic factors.

Several potential limitations in our study should be pointed out. First, we did not investigate the IncRNA ANRIL expression in the study groups, and therefore were unable to determine a link between the SNPs and transcription of IncRNA ANRIL. Second, the small sample size and inclusion of only one Iranian population can decrease the statistical power of our study, thus we recommended replication studies with a larger sample size to assess the involvement of $A N R / L$ polymorphisms in AR pathogenesis. Third, the present study did not cover all of the genetic polymorphisms on $A N R I L$, and thus, further studies are required to identify potential causative variants. Fourth, the distribution of genotypes of rs 10757278 was not in accordance with HWE in the study groups, probably due to inbreeding or small population sizes. Therefore, the obtained results should be interpreted with caution.

\section{Conclusion}

In conclusion, our results represented no significant difference between ANRIL gene rs10757278 and rs1333048 polymorphisms with AR susceptibility. Further studies are needed to indicate the possible contribution of $A N R I L$ gene SNPs as a genetic risk factor in the pathogenesis of AR.

\section{Declarations}

\section{Acknowledgments}

The authors thank the Vice Chancellor for Research and Technology of Kermanshah University of Medical Sciences for their financial support. This work was part of a project supported by the Research Council of Kermanshah University of Medical Sciences. 


\section{Authorship contributions}

A. R .M. was involved in the concept and design of the study. Sara F. drafted the manuscript. All authors were involved in data collection, analysis, and interpretation and approved the final manuscript.

\section{Funding}

This study was supported by the Research Administration Department of Kermanshah University of Medical Sciences, Kermanshah, Iran [ A.R.M., Grant Number: KUMS 3010385].

\section{Conflict of interest}

The authors declare that they have no conflicts of interest regarding this manuscript.

\section{References}

1. Abd-Elmawla, M.A., Fawzy, M.W., Rizk, S.M., and Shaheen, A.A. (2018). Role of long non-coding RNAs expression (ANRIL, NOS3-AS, and APOA1-AS) in development of atherosclerosis in Egyptian systemic lupus erythematosus patients. Clinical Rheumatology 37, 3319-3328.

2. Broide, D.H. (2010). Allergic rhinitis: Pathophysiology. In 5.

3. Chen, S., Zhong, H., Wang, Y., Wang, Z., Liang, X., Li, S., Li, Z., Yu, Z., Li, L., and Yi, G. (2019). The clinical significance of long non-coding RNA ANRIL level in diabetic retinopathy. Acta diabetologica, $1-10$.

4. DeVries, A., and Vercelli, D. (2015). Epigenetics in allergic diseases. Current opinion in pediatrics 27, 719.

5. Flores-Concha, M., and Oñate, Á.A. (2020). Long Non-coding RNAs in the Regulation of the Immune Response and Trained Immunity. Frontiers in Genetics 11.

6. Gao, F., Cai, Y., Kapranov, P., and Xu, D. (2020). Reverse-genetics studies of IncRNAs-what we have learnt and paths forward. Genome Biology 21,1-23.

7. Ghafouri-Fard, S., Shoorei, H., Taheri, M., and Sanak, M. (2020). Emerging role of non-coding RNAs in allergic disorders. Biomedicine \& Pharmacotherapy 130,110615.

8. Hariri, F.Y., Salahshourifar, I., and Karizi, S.Z. (2020). Association between coronary artery disease and rs10757278 and rs1333049 polymorphisms in 9p21 locus in Iran. Reports of Biochemistry \& Molecular Biology 9, 58 .

9. Harismendy, O., Notani, D., Song, X., Rahim, N.G., Tanasa, B., Heintzman, N., Ren, B., Fu, X.-D., Topol, E.J., and Rosenfeld, M.G. (2011). 9p21 DNA variants associated with coronary artery disease impair interferon-y signalling response. Nature 470, 264-268.

10. Hori, D., Dunkerly-Eyring, B., Nomura, Y., Biswas, D., Steppan, J., Henao-Mejia, J., Adachi, H., Santhanam, L., Berkowitz, D.E., and Steenbergen, C. (2017). miR-181b regulates vascular stiffness age dependently in part by regulating TGF- $\beta$ signaling. PloS one 12, e0174108. 
11. Huo, X., Zhang, K., Yi, L., Mo, Y., Liang, Y., Zhao, J., Zhang, Z., Xu, Y., and Zhen, G. (2016). Decreased epithelial and plasma miR-181b-5p expression associates with airway eosinophilic inflammation in asthma. Clinical \& Experimental Allergy 46, 1281-1290.

12. Kabesch, M., and Tost, J. (2020). Recent findings in the genetics and epigenetics of asthma and allergy. (Springer), pp. 1-18.

13. Khorshidi, H.R., Taheri, M., Noroozi, R., Sarrafzadeh, S., Sayad, A., and Ghafouri-Fard, S. (2017). ANRIL genetic variants in Iranian breast cancer patients. Cell Journal (Yakhteh) 19, 72.

14. Li, L., Zhang, S., Jiang, X., Liu, Y., Liu, K., and Yang, C. (2018). MicroRNA-let-7e regulates the progression and development of allergic rhinitis by targeting suppressor of cytokine signaling 4 and activating Janus kinase 1/signal transducer and activator of transcription 3 pathway. Experimental and therapeutic medicine $15,3523-3529$.

15. Lu, W.-H., Zhang, W.-Q., Zhao, Y.-J., Gao, Y.-T., Tao, N., Ma, Y.-T., Liu, J.-W., and Wulasihan, M. (2020). Case-Control Study on the Interaction Effects of rs10757278 Polymorphisms at 9p21 Locus and Traditional Risk Factors on Coronary Heart Disease in Xinjiang, China. Journal of Cardiovascular Pharmacology 75, 439-445.

16. Mehta-Mujoo, P., Cunliffe, H., Hung, N.A., and Slatter, T.L. (2019). Long non-coding RNA ANRIL in the nucleus associates with periostin expression in breast cancer. Frontiers in oncology 9, 885 .

17. Mims, J.W. (2014). Epidemiology of allergic rhinitis. In S2. (Wiley Online Library), pp. S18-S20.

18. Mollahosseini, M., Rahimi, M.H., Yekaninejad, M.S., Maghbooli, Z., and Mirzaei, K. (2020). Dietary patterns interact with chromosome 9p21 rs1333048 polymorphism on the risk of obesity and cardiovascular risk factors in apparently healthy Tehrani adults. European Journal of Nutrition 59 , $35-43$.

19. Mulrane, L., McGee, S.F., Gallagher, W.M., and O'Connor, D.P. (2013). miRNA dysregulation in breast cancer. Cancer research 73, 6554-6562.

20. Niemiec, P., Gorczynska-Kosiorz, S., Iwanicki, T., Krauze, J., Trautsolt, W., Grzeszczak, W., Bochenek, A., and Zak, I. (2012). The rs10757278 polymorphism of the 9p21. 3 locus is associated with premature coronary artery disease in Polish patients. Genetic testing and molecular biomarkers 16, 1080-1085.

21. Qian, X., Shi, S., and Zhang, G. (2019). Long non-coding RNA antisense non-coding RNA in the INK4 locus expression correlates with increased disease risk, severity, and inflammation of allergic rhinitis. Medicine 98.

22. Qin, Z., and Liu, D. (2019). Circulating IncRNA ANRIL level positively correlates with disease risk, severity, inflammation level and poor prognosis of coronary artery disease. Biomedical Research (0970-938X) 30.

23. Rakhshan, A., Zarrinpour, N., Moradi, A., Ahadi, M., Omrani, M.D., Ghafouri-Fard, S., and Taheri, M. (2020). Genetic variants within ANRIL (antisense non coding RNA in the INK4 locus) are associated with risk of psoriasis. International Immunopharmacology 78, 106053.

24. Rezazadeh, M., Gharesouran, J., Moradi, M., Noroozi, R., Omrani, M.D., Taheri, M., and Ghafouri-Fard, S. (2018). Association study of ANRIL genetic variants and multiple sclerosis. Journal of Molecular 
Neuroscience 65, 54-59.

25. Suleiman, A.A., Muhsin, H., Abdulkareem, R.A., and Abed, F.A. (2019). Association study of two single nucleotide polymorphisms rs10757278 and rs1333049 with atherosclerosis, a case-control study from Iraq. Molecular Biology Research Communications 8, 99.

26. Taheri, M., Pouresmaeili, F., Omrani, M.D., Habibi, M., Sarrafzadeh, S., Noroozi, R., Rakhshan, A., Sayad, A., and Ghafouri-Fard, S. (2017). Association of ANRIL gene polymorphisms with prostate cancer and benign prostatic hyperplasia in an Iranian population. Biomarkers in medicine 11, 413422.

27. Varshney, J., and Varshney, H. (2015). Allergic rhinitis: an overview. Indian Journal of Otolaryngology and Head \& Neck Surgery 67, 143-149.

28. Wang, X., Du, K., She, W., Ouyang, Y., Sima, Y., Liu, C., and Zhang, L. (2018). Recent advances in the diagnosis of allergic rhinitis. Expert review of clinical immunology 14, 957-964.

29. Wee, J.H., Zhang, Y.-L., Rhee, C.-S., and Kim, D.-Y. (2017). Inhibition of allergic response by intranasal selective NF-KB decoy oligodeoxynucleotides in a murine model of allergic rhinitis. Allergy, asthma \& immunology research 9, 61-69.

30. Wei, J.-W., Huang, K., Yang, C., and Kang, C.-S. (2017). Non-coding RNAs as regulators in epigenetics. Oncology reports $37,3-9$.

31. Wheatley, L.M., and Togias, A. (2015). Allergic rhinitis. New England Journal of Medicine 372, 456463.

32. Zhang, X., Wang, W., Zhu, W., Dong, J., Cheng, Y., Yin, Z., and Shen, F. (2019). Mechanisms and functions of long non-coding RNAs at multiple regulatory levels. International journal of molecular sciences 20, 5573 .

33. Zhou, X., Han, X., Wittfeldt, A., Sun, J., Liu, C., Wang, X., Gan, L.-M., Cao, H., and Liang, Z. (2016). Long non-coding RNA ANRIL regulates inflammatory responses as a novel component of NF-KB pathway. RNA biology $13,98-108$.

34. Zuo, Y., and Xu, H. (2020). Involvement of long noncoding RNAs in the pathogenesis of autoimmune diseases. Journal of Translational Autoimmunity, 100044.

\section{Figures}




\section{GG GG AA AG AG AA AG AG AA AG}

$\begin{array}{lll}443 b p & 443 b p & 443 b p \\ & 262 b p & 262 b p \\ & & 236 b p\end{array}$

\section{Figure 1}

products of IncRNA ANRIL rs10757278 A>G polymorphism on $2 \%$ agarose gel.

Homozygotes wild AA genotype (443bp,262bp); heterozygous AG genotype (443bp, 262bp and 236bp); mutant GG genotype (443bp, 236bp); NG (negative control). 


\section{AC CC AA Ng AA AC CC AC AA AA AC AC}

$$
\frac{381}{\frac{252}{184}}-\frac{381}{184}--\frac{381}{-52}=---
$$

\section{Figure 2}

products of IncRNA ANRIL rs1333048 A> C polymorphism on 2\% agarose gel.

Homozygotes wild AA genotype (381bp,184bp); heterozygous AC genotype (381bp, 252bp and 184bp); mutant CC genotype (381bp, 184bp); NG (negative control). 